\title{
Systematic Errors and Location Accuracy in Wireless Networks
}

\author{
Harri Saarnisaari and Timo Bräysy \\ Centre for Wireless Communications, University of Oulu, P.O. Box 4500, 90014 Oulu, Finland
}

Received 13 May 2005; Revised 16 March 2006; Accepted 23 March 2006

\begin{abstract}
Wireless systems already provide time delay and signal strength measurements and the future may see antenna arrays that provide directional information. All these may be used for positioning. Although the statistical accuracy of different positioning methods is well studied, the systematic error effects, which arise, for example, from errors in sensor (node) location, network synchronization, or the path loss model, are not. This study fills this gap providing a unified error-propagation-law-based tool to analyze measurement and systematic error effects. The considered positioning systems, which are compared based on the developed framework, are the hyperbolic (time-delay-based), direction finding (DF), received signal strength (RSS), and relative RSS (RRSS) location systems. The obtained analytical results verify our intuitive expectations; the hyperbolic methods are sensitive to errors in network synchronization, RRRS methods to channel modelling errors, whereas DF methods are rather insensitive to systematic errors. However, the bias of DF methods is at its largest if the sensor location error is perpendicular to the line joining the sensor and the source. If the methods are compared based on overall accuracy, hyperbolic methods may be preferred in large sized networks, whereas the DF and RRSS methods may provide better accuracy in small sized networks. However, RRSS systems require a dense network in order to provide reliable results.
\end{abstract}

Copyright (c) 2006 H. Saarnisaari and T. Bräysy. This is an open access article distributed under the Creative Commons Attribution License, which permits unrestricted use, distribution, and reproduction in any medium, provided the original work is properly cited.

\section{INTRODUCTION}

Not just are location-based services becoming more popular in wireless systems but location is needed also in wireless sensing applications $[1,2]$. A fundamental question in location is what is the attainable location accuracy and what is required to obtain it; whether some methods can offer sufficient accuracy with lower complexity and, possibly, with simpler installation than other ones. Positioning accuracy is affected by the measurement accuracy and systematic errors. A systematic error may be defined as an error that is not determined by chance but is introduced by an inaccuracy (as of observation or measurement) inherent in the system (MerriamWebster On-Line http://www.m-w.com/). Therefore, systematic errors, in contrast to random ones, are reproducible inaccuracies that are consistently in the same direction. In this work systematic errors include errors in wireless node positions, network synchronization, the propagation path loss model, and so forth.

The effects of measurement accuracy are well studied, see, for example, [3-12], but systematic error effects are not. They are often just discussed, see, for example, [11].

The measurement error effects are usually approximated using the Cramér-Rao lower bound (CRLB) as in the above-mentioned references. It is used also in [13] to study the effects of sensor location uncertainties assuming sensor locations are random variables with the nominal locations as means. Reference [14] studied aperture error effects on frequency-based emitter location using perturbation analysis instead of the usual Taylor series approach [4] which yields the CRLB in the Gaussian case. The Taylor series approach is used in this paper to provide a unified tool to analyze both the measurement and systematic error effects on the location accuracy. This approach was suggested in [4], but the authors have not seen any attempts to apply it. The method is equal to the law of propagation of uncertainty (see http://physics.nist.gov/cuu/Uncertainty/, the web page of Physics Laboratory of National Institute of Standards and Technology) [15].

We briefly explain the analysis framework in Section 2 and apply it to the analysis of the location accuracy of hyperbolic (time-delay-(TD) based), direction finding (DF) and received signal strength (RSS) location systems in Sections 3, 4 , and 5 , respectively. The location methods are compared in Section 6. The proposed analysis tool can also be used to analyze other possible location systems, for example, hybrid systems where time, angle, and strength measurements are used for positioning. In this sense the paper serves as an example 
of the usage of the method. Hybrid methods and the CRLB are discussed in [16].

We have some expectations about how sensitive these location systems are to systematic errors, see, for example, [11]. Hyperbolic systems are expected to be sensitive to network synchronization errors, RSS methods to path loss modelling errors, whereas DF methods are rather insensitive to systematic errors. However, it is expected that in DF methods the resulting error is largest when the sensor location error is perpendicular to the line joining the sensor and the source. Luckily, we can confirm these expectations analytically and also provide a tool to calculate numerical values for the resulting location errors.

\section{ANALYSIS TOOL}

Let $\mathbf{x}$ be the estimator of the actual location $\mathbf{x}^{0}$ either in two or three dimensions. The bias is $\mathbf{b}=\mathrm{E}\left\{\mathbf{x}-\mathbf{x}^{0}\right\}$, the covariance $\mathrm{C}=\mathrm{E}\left\{(\mathbf{x}-\mathrm{E}\{\mathbf{x}\})(\mathbf{x}-\mathrm{E}\{\mathbf{x}\})^{\mathrm{T}}\right\}$, and the total mean-squared error $\mathbf{V}=\mathrm{E}\left\{\left(\mathbf{x}-\mathbf{x}^{0}\right)\left(\mathbf{x}-\mathbf{x}^{0}\right)^{\mathrm{T}}\right\}=\mathbf{b b}^{\mathrm{T}}+\mathbf{C}$. Therefore, the analysis of the bias and covariance suffices to determine the accuracy of location systems.

Let the measurements without uncertainties be defined as $r_{i}^{0}=f_{i}\left(\mathbf{x}^{0}, \mathbf{q}^{0}\right)$, or in a vector form

$$
\mathbf{r}^{0}=\mathbf{f}\left(\mathbf{x}^{0}, \mathbf{q}^{0}\right)
$$

where $\mathbf{q}$, in general, denotes the system parameters that affect the measurement model and $\mathbf{q}^{0}$ denotes the actual system parameters. The model is, in general, nonlinear. Therefore, it is linearized using the first two terms of the Taylor series of $\mathbf{r}=\mathbf{f}(\mathbf{x}, \mathbf{q})$ around $\mathbf{x}^{0}$ and $\mathbf{q}^{0}$. Assuming that $\mathbf{x}$ and $\mathbf{q}$ are close to the actual values and that the cross terms between measurement and systematic errors are insignificant ${ }^{1}$ (or "independent"), it follows that $[4,15,17]$

$$
\mathbf{r} \approx \mathbf{f}\left(\mathbf{x}^{0}, \mathbf{q}^{0}\right)+J_{\mathbf{x}}\left(\mathbf{x}^{0}, \mathbf{q}^{0}\right)\left(\mathbf{x}-\mathbf{x}^{0}\right)+J_{\mathbf{q}}\left(\mathbf{x}^{0}, \mathbf{q}^{0}\right)\left(\mathbf{q}-\mathbf{q}^{0}\right),
$$

where $J_{\mathbf{x}}(\mathbf{x}, \mathbf{z})$ and $J_{\mathbf{q}}(\mathbf{x}, \mathbf{q})$ are the Jacobians of $\mathbf{f}(\mathbf{x}, \mathbf{q})$ with respect to $\mathbf{x}$ and $\mathbf{q}$. The first two terms are those used in [4] to solve the measurement error effects problem. In [13] this insignificancy assumption is not made, but neither are the results so simply expressed as ours. Solving this linear equation for $\mathbf{x}-\mathbf{x}^{0}$ gives

$$
\begin{aligned}
\mathbf{x}-\mathbf{x}^{0} \approx & \left(J_{\mathbf{x}}^{\mathrm{T}}\left(\mathbf{x}^{0}, \mathbf{q}^{0}\right) J_{\mathbf{x}}\left(\mathbf{x}^{0}, \mathbf{q}^{0}\right)\right)^{-1} J_{\mathbf{x}}^{\mathrm{T}}\left(\mathbf{x}^{0}, \mathbf{q}^{0}\right) \\
& \times\left(\mathbf{r}-\mathbf{f}\left(\mathbf{x}^{0}, \mathbf{q}^{0}\right)-J_{\mathbf{q}}\left(\mathbf{x}^{0}, \mathbf{q}^{0}\right)\left(\mathbf{q}-\mathbf{q}^{0}\right)\right) .
\end{aligned}
$$

In reality, the measurements $\mathbf{r}$ are disturbed by measurement errors e such that $\mathbf{r}=\mathbf{r}^{0}+\mathbf{e}$. Substituting this into (3) yields

$$
\begin{aligned}
\mathbf{x}-\mathbf{x}^{0} \approx & \left(J_{\mathbf{x}}^{\mathrm{T}}\left(\mathbf{x}^{0}, \mathbf{q}^{0}\right) J_{\mathbf{x}}\left(\mathbf{x}^{0}, \mathbf{q}^{0}\right)\right)^{-1} J_{\mathbf{x}}^{\mathrm{T}}\left(\mathbf{x}^{0}, \mathbf{q}^{0}\right) \\
& \times\left(\mathbf{e}-J_{\mathbf{q}}\left(\mathbf{x}^{0}, \mathbf{q}^{0}\right)\left(\mathbf{q}-\mathbf{q}^{0}\right)\right) .
\end{aligned}
$$

\footnotetext{
1 This also simplifies our analysis; the another reason. See, for further discussions, http://physics.nist.gov/cuu/Uncertainty/.
}

This is the result that allows us to analyze the bias and covariance of the positioning error. The measurement errors e are typically modelled as zero mean Gaussian random variables with the covariance $\mathbf{C}_{\mathrm{e}}$. Alternatively, the measurement errors may contain bias $\mathbf{b}_{\mathbf{e}}$. This bias may arise, for example, from calibration errors. Systematic errors are most often modelled as deterministic variables, but can also be modelled as random ones, in which case one obtains an average performance over different possible system errors. Herein, the deterministic viewpoint is used.

The term

$$
\mathbf{b}_{\mathbf{r}}=J_{\mathbf{q}}\left(\mathbf{x}^{0}, \mathbf{q}^{0}\right)\left(\mathbf{q}-\mathbf{q}^{0}\right)
$$

presents the bias in the measurements due to the systematic errors. If there exists an additional measurement bias $\mathbf{b}_{\mathbf{e}}$ through calibration errors, it can be added to the measurement bias (5). The location bias resulting from (4) and the definition (5) is

$$
\mathbf{b}=-\left(J_{\mathbf{x}}^{\mathrm{T}}\left(\mathbf{x}^{0}, \mathbf{q}^{0}\right) J_{\mathbf{x}}\left(\mathbf{x}^{0}, \mathbf{q}^{0}\right)\right)^{-1} J_{\mathbf{x}}^{\mathrm{T}}\left(\mathbf{x}^{0}, \mathbf{q}^{0}\right) \mathbf{b}_{\mathbf{r}} .
$$

The positioning error covariance becomes

$$
\begin{aligned}
\mathbf{C}= & \left(J_{\mathbf{x}}^{\mathrm{T}}\left(\mathbf{x}^{0}, \mathbf{q}^{0}\right) J_{\mathbf{x}}\left(\mathbf{x}^{0}, \mathbf{q}^{0}\right)\right)^{-1} J_{\mathbf{x}}^{\mathrm{T}}\left(\mathbf{x}^{0}, \mathbf{q}^{0}\right) \mathbf{C}_{\mathbf{e}} \\
& \times J_{\mathbf{x}}\left(\mathbf{x}^{0}, \mathbf{q}^{0}\right)\left(J_{\mathbf{x}}^{\mathrm{T}}\left(\mathbf{x}^{0}, \mathbf{q}^{0}\right) J_{\mathbf{x}}\left(\mathbf{x}^{0}, \mathbf{q}^{0}\right)\right)^{-1} .
\end{aligned}
$$

It is shown in the appendix that $\mathbf{C}$ can be upper bounded by

$$
\mathbf{C}_{u}=n \sigma_{e}^{2}\left(J_{\mathbf{x}}^{\mathrm{T}}\left(\mathbf{x}^{0}, \mathbf{q}^{0}\right) J_{\mathbf{x}}\left(\mathbf{x}^{0}, \mathbf{q}^{0}\right)\right)^{-1}
$$

where $n$ is the number of measurements (the dimension of r) and $\sigma_{e}^{2}$ is the maximum (diagonal) element of $\mathbf{C}_{\mathbf{e}}$. The upper bound is in the sense that the matrix difference $\mathbf{C}_{u}-\mathbf{C}$ is positive semidefinite. It is also shown in the appendix that a tighter upper bound and a lower bound can be obtained by substituting $n \sigma_{e}^{2}$ by the maximum and minimum eigenvalues of $\mathbf{C}_{e}$, respectively. For a diagonal (or nearly so) $\mathbf{C}_{e}$, this is equivalent to choosing the maximum and minimum variances.

It can be concluded that the mean-squared error is upper bounded by

$$
\mathbf{V}_{u}=n\left(b^{2}+\sigma_{e}^{2}\right)\left(J_{\mathbf{x}}^{\mathrm{T}}\left(\mathbf{x}^{0}, \mathbf{q}^{0}\right) J_{\mathbf{x}}\left(\mathbf{x}^{0}, \mathbf{q}^{0}\right)\right)^{-1},
$$

where $b$ is the maximum element of $\mathbf{b}_{\mathbf{r}}^{\mathrm{T}} \mathbf{b}_{\mathbf{r}}$. Equation (9) forms a simple way to find an approximative performance of a location system. This is done selecting the maximum single measurement error bias $b$ and maximum estimation error variance $\sigma_{e}^{2}$ (as in the upper bound above) and multiplying these by the term $\mathbf{Q}=\left(J_{\mathbf{x}}^{\mathrm{T}}\left(\mathbf{x}^{0}, \mathbf{q}^{0}\right) J_{\mathbf{x}}\left(\mathbf{x}^{0}, \mathbf{q}^{0}\right)\right)^{-1}$. The term $\mathbf{Q}$ presents effects of the sensor geometry to the location accuracy and may be called the geometric dilution of precision (GDOP) [4]. Reference [4] gives results related to it for hyperbolic and direction finding location systems and also discusses other definitions for the location error. 
It is also possible to solve the unknown location using the weighted least squares $[3,4]$. The resulting location error is

$$
\begin{aligned}
\mathbf{x}-\mathbf{x}^{0} \approx & \left(J_{\mathbf{x}}^{\mathrm{T}}\left(\mathbf{x}^{0}, \mathbf{q}^{0}\right) \mathbf{W} J_{\mathbf{x}}\left(\mathbf{x}^{0}, \mathbf{q}^{0}\right)\right)^{-1} J_{\mathbf{x}}^{\mathrm{T}}\left(\mathbf{x}^{0}, \mathbf{q}^{0}\right) \\
& \times \mathbf{W}\left(\mathbf{e}-J_{\mathbf{q}}\left(\mathbf{x}^{0}, \mathbf{q}^{0}\right)\left(\mathbf{q}-\mathbf{q}^{0}\right)\right),
\end{aligned}
$$

where $\mathbf{W}$ is a positive definite weighting matrix. It can be an identity matrix, but the optimal choice is $\mathbf{W}=\mathbf{C}_{\mathbf{e}}$ if the covariance $\mathbf{C}_{\mathbf{e}}$ is known (or estimated somehow) $[3,4]$. In this case the position error covariance becomes $\mathbf{C}=\left(J_{\mathbf{x}}^{\mathrm{T}}\left(\mathbf{x}^{0}, \mathbf{q}^{0}\right) \mathbf{C}_{\mathrm{e}}^{-1} J_{\mathbf{x}}\left(\mathbf{x}^{0}, \mathbf{q}^{0}\right)\right)^{-1}$. This is equal to the wellknown CRLB (see, e.g., $[3,10]$ ) if the estimation error is Gaussian. It can be shown, as in the appendix, that $\left(J_{\mathbf{x}}^{\mathrm{T}}\left(\mathbf{x}^{0}, \mathbf{q}^{0}\right) \mathbf{C}_{\mathbf{e}}^{-1} J_{\mathbf{x}}\left(\mathbf{x}^{0}, \mathbf{q}^{0}\right)\right)^{-1}$ is upper and lower bounded by $d\left(J_{\mathbf{x}}^{\mathrm{T}}\left(\mathbf{x}^{0}, \mathbf{q}^{0}\right) J_{\mathbf{x}}\left(\mathbf{x}^{0}, \mathbf{q}^{0}\right)\right)^{-1}$, where $d$ is the maximum or minimum eigenvalue of $\mathbf{C}_{e}$, respectively. If $\mathbf{C}_{\mathbf{e}}$ is diagonal, maximum and minimum variances may be used to obtain the bounds.

The main aim of this paper is to investigate the bias $\mathbf{b}_{\mathbf{r}}$ and how it translates to location errors through the GDOP in different location systems.

\section{HYPERBOLIC LOCATION}

Hyperbolic location systems use differences of times-ofarrival (TOAs) measured either directly [18] or subtracting measured TOAs [4]. Therefore, they may be called time difference (TD) location systems. The methods require that the sensor or base station network is synchronized or sensors/base stations have known relations between their clocks. Let the transmission time be denoted as $t_{0}$. The signal pass the distance $d_{i}^{0}=\left\|\mathbf{x}^{0}-\mathbf{x}_{i}^{0}\right\|$, where $\|\cdot\|$ denotes the Euclidean vector norm, between the emitter and the sensor $i$ at $\mathbf{x}_{i}$ at time $\tau_{i}$. The receiver therefore observes the signal at time $t_{i}=t_{0}+\tau_{i}+\delta_{i}$, where $\delta_{i}$ denotes the network synchronization error, that is, the time difference to the common time of the sensor network. Let sensor $j$ be a reference sensor. Subtracting $t_{j}$ from the other measurements and multiplying the results with the propagation speed of the signal $c$, the measurements of the hyperbolic location systems are modelled as

$$
r_{i}=c\left(t_{i}-t_{j}\right)=\left\|\mathbf{x}-\mathbf{x}_{i}\right\|-\left\|\mathbf{x}-\mathbf{x}_{j}\right\|+c\left(\delta_{i}-\delta_{j}\right)+e_{i}
$$

If there are $N$ sensors, then there are $N-1$ measurements, the minimum possible number being $N=D+1$, where $D$ is the dimension of the location problem, that is, two or three.

Clearly, the system parameters

$$
\mathbf{q}=\left[\begin{array}{llllll}
\mathbf{x}_{1}^{\mathrm{T}} & \cdots & \mathbf{x}_{N}^{\mathrm{T}} & \delta_{1} & \cdots & \delta_{N}
\end{array}\right]^{\mathrm{T}}
$$

include the locations of the sensors $\mathbf{x}_{i}$ and the clock errors $\delta_{i}$. The actual sensor locations are $\mathbf{x}_{i}^{0}$. The assumed sensor locations may differ from the actual sensor positions by $\delta_{\mathbf{x}_{i}}$. The desired value $\delta_{i}^{0}$ for the clock errors is naturally zero. For brevity, let $J_{\mathbf{x}}=J_{\mathbf{x}}\left(\mathbf{x}^{0}, \mathbf{q}^{0}\right)$. Then, the Jacobian with respect to the unknown location is

$$
J_{\mathbf{x}}=\left[\begin{array}{c}
\frac{\left(\mathbf{x}^{0}-\mathbf{x}_{1}^{0}\right)^{\mathrm{T}}}{d_{1}^{0}}-\frac{\left(\mathbf{x}^{0}-\mathbf{x}_{j}^{0}\right)^{\mathrm{T}}}{d_{j}^{0}} \\
\vdots \\
\frac{\left(\mathbf{x}^{0}-\mathbf{x}_{j-1}^{0}\right)^{\mathrm{T}}}{d_{j-1}^{0}}-\frac{\left(\mathbf{x}^{0}-\mathbf{x}_{j}^{0}\right)^{\mathrm{T}}}{d_{j}^{0}} \\
\frac{\left(\mathbf{x}^{0}-\mathbf{x}_{j+1}^{0}\right)^{\mathrm{T}}}{d_{j+1}^{0}}-\frac{\left(\mathbf{x}^{0}-\mathbf{x}_{j}^{0}\right)^{\mathrm{T}}}{d_{j}^{0}} \\
\frac{\left(\mathbf{x}^{0}-\mathbf{x}_{N}^{0}\right)^{\mathrm{T}}}{d_{N}^{0}}-\frac{\left(\mathbf{x}^{0}-\mathbf{x}_{j}^{0}\right)^{\mathrm{T}}}{d_{j}^{0}}
\end{array}\right] .
$$

The $i$ th element of the bias $\mathbf{b}_{\mathbf{r}}$ is

$$
\left[\mathbf{b}_{\mathbf{r}}\right]_{i}=\frac{-\left(\mathbf{x}^{0}-\mathbf{x}_{i}^{0}\right)^{\mathrm{T}} \delta_{\mathbf{x}_{i}}}{d_{i}^{0}}+\frac{\left(\mathbf{x}^{0}-\mathbf{x}_{j}^{0}\right)^{\mathrm{T}} \delta_{\mathbf{x}_{j}}}{d_{j}^{0}}+c\left(\delta_{i}-\delta_{j}\right) .
$$

The terms may be contrastive (to "opposite directions") resulting in a small bias. The terms may, as well, be restorative (to "equal directions") resulting in a large bias. The clock error term, the last term in (14), attains its maximum if the clock errors are to opposite directions and minimum if the clock errors are to equal direction. In order to see the magnitude of this bias, notice that if the clock error $\delta_{i}-\delta_{j}$ is $3 \mathrm{~ns}$, $1 \mu \mathrm{s}$, or $1 \mathrm{~ms}$ and the signal is a radio signal, then the caused biases are of order $1 \mathrm{~m}, 300 \mathrm{~m}$, and $300 \mathrm{~km}$, respectively. The clock errors may therefore be rather fatal to the accuracy of hyperbolic location systems and clearly show why very accurate network synchronization is required in these systems.

Due to the Schwarz inequality the first two terms in (14) are bounded as

$$
\frac{\left\|\left(\mathbf{x}^{0}-\mathbf{x}_{i}^{0}\right)^{\mathrm{T}} \delta_{\mathbf{x}_{i}}\right\|}{d_{i}^{0}} \leq \frac{1}{d_{i}^{0}}\left\|\left(\mathbf{x}^{0}-\mathbf{x}_{i}^{0}\right)\right\|\left\|\delta_{\mathbf{x}_{i}}\right\|=\left\|\delta_{\mathbf{x}_{i}}\right\|,
$$

with equality if and only if $\delta_{\mathbf{x}_{i}}=\alpha\left(\mathbf{x}^{0}-\mathbf{x}_{i}^{0}\right)$, where $\alpha$ is a constant. Consequently, the errors through the sensor locations are at maximum if $\delta_{\mathbf{x}_{i}}$ are parallel to $\mathbf{x}^{0}-\mathbf{x}_{i}^{0}$. These errors vanish if $\delta_{\mathbf{x}_{i}}$ and $\mathbf{x}^{0}-\mathbf{x}_{i}^{0}$ are orthogonal. Indeed, this result approximates the actual situation as shown in Figure 1. The bias due to sensor location errors are not necessarily very large since the sensors (or base stations) can typically be set within a few meters. However, the required sensor location accuracy depends on the demands of the application. The sensor location accuracy cannot be larger than the accuracy required from the system. Otherwise, the bias through the sensor location accuracy may dominate the error budget.

It is readily understood that these bias results could easily be derived without the used technique, just using logical reasoning. However, the technique allows us to determine also the covariance. As a consequence, the used technique offers a unified method to analyze the accuracy of location systems. 


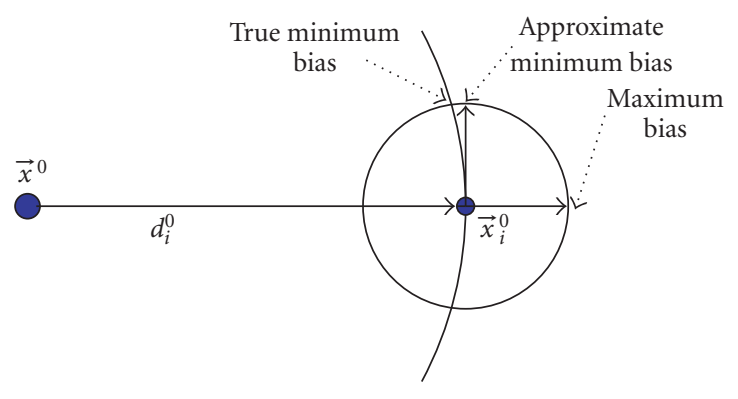

FIgURE 1: Illustration of the true and approximate bias through the sensor location error.

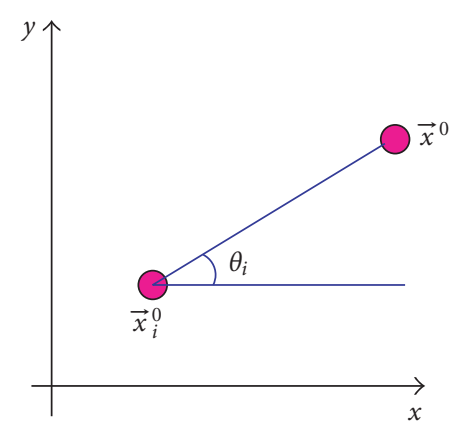

Figure 2: The definition of the DOA measurements.

It is well known that the TOA estimation accuracy $\sigma_{\tau}$ is proportional to the signal-to-noise ratio $\gamma$ and the signal bandwidth $W$, see, for example, [4], such that

$$
\sigma_{\tau} \propto \frac{1}{W \sqrt{\gamma}} .
$$

In this light, wideband systems like ultra-wideband (UWB) and wideband code division multiple access (WCDMA) are better for hyperbolic location than the GSM system that uses a rather narrowband signal. This is especially true if high accuracy is required. One problem with TOA measurements is the absence of the line-of-sight (LOS) signal which is tacitly assumed in the previous results. Instead, the TOA may be measured from a reflected signal which causes additional bias. One solution to this non-LOS situation is presented in [19].

\section{DIRECTION FINDING LOCATION}

The direction finding (DF) location system is considered in a two-dimensional case. The measured direction of arrival (DOA) is defined in Figure 2. It follows that the measurements are modelled as [4]

$$
r_{i}=\arctan \frac{y-y_{i}}{x-x_{i}}+e_{i}
$$

where $\mathbf{x}=\left[\begin{array}{ll}x & y\end{array}\right]^{\mathrm{T}}$ and $\mathbf{x}_{i}=\left[\begin{array}{ll}x_{i} & y_{i}\end{array}\right]^{\mathrm{T}}$. Thus, the parameter vector $\mathbf{q}=\left[\begin{array}{lll}\mathbf{x}_{1}^{\mathrm{T}} & \cdots & \mathbf{x}_{N}^{\mathrm{T}}\end{array}\right]^{\mathrm{T}}$ involves only the sensor locations.
The Jacobian becomes

$$
J_{\mathbf{x}}=\left[\begin{array}{cc}
\frac{-\left(y^{0}-y_{1}^{0}\right)}{\left(d_{1}^{0}\right)^{2}} & \frac{\left(x^{0}-x_{1}^{0}\right)}{\left(d_{1}^{0}\right)^{2}} \\
\vdots & \vdots \\
\frac{-\left(y^{0}-y_{N}^{0}\right)}{\left(d_{N}^{0}\right)^{2}} & \frac{\left(x^{0}-x_{N}^{0}\right)}{\left(d_{N}^{0}\right)^{2}}
\end{array}\right] .
$$

The ith bias term (in radians) is

$$
\left[\mathbf{b}_{\mathbf{r}}\right]_{i}=\left[\frac{\left(y^{0}-y_{1}^{0}\right)}{\left(d_{i}^{0}\right)^{2}} \frac{-\left(x^{0}-x_{1}^{0}\right)}{\left(d_{i}^{0}\right)^{2}}\right] \delta_{\mathbf{x}_{i}} .
$$

Let $\mathbf{a}=\left[\left(y^{0}-y_{i}^{0}\right)-\left(x^{0}-x_{1}^{0}\right)\right]^{\mathrm{T}}$. Due to the Schwartz inequality the maximum bias $\left\|\delta_{\mathbf{x}_{i}}\right\| / d_{i}^{0}$ occurs if $\delta_{\mathbf{x}_{i}}$ is parallel with a. Since $\mathbf{x}^{0}-\mathbf{x}_{i}^{0}$ is orthogonal to $\mathbf{a}$, the maximum measurement bias occurs if the location error is orthogonal to the line joining the sensor and the emitter. This is obvious also from Figure 3. The measurement bias vanishes if $\delta_{\mathbf{x}_{i}}$ is orthogonal to a, or, equally, parallel with the line joining the emitter and the sensor as can be observed also from Figure 3.

The maximum bias $\mathbf{b}_{\mathbf{r}}$ decreases as the distance between the emitter and the sensor increases, as can also be logicaly concluded. The measurement bias may be significant if the sensor location accuracy is not small compared to the emitter-sensor distance. The accuracy of the DOA estimator can be well approximated by the Cramér-Rao bound at sufficiently high SNR values. The bound for uniform linear arrays can be found from [20]. At large emitter sensor distances the measurement error dominates the error budget. As a conclusion, it was shown that the DF location methods are rather insensitive to systematic errors.

\section{SIGNAL STRENGTH LOCATION}

In received signal strength (RSS) location systems the path loss is assumed to have a known relation with the distance. However, there are several possible path loss models and, consequently, several possible analysis. In this paper a simple model is used, but the presented technique can be applied to any other model; a possible model is discussed in [11]. Let the measurements, the received signal power, be modelled simply as

$$
r_{i}=P_{r_{i}}=P_{t}\left(\frac{c}{4 \pi f_{0} d_{0}}\right)^{2}\left(\frac{f_{0}}{f}\right)^{\beta}\left(\frac{d_{0}}{d}\right)^{\alpha}+e_{i},
$$

where $d_{0}$ and $f_{0}$ are the reference distance and frequency, respectively, and the frequency $f$ is the known factor while the unknown assumed factors are $\alpha$ and $\beta$, which typically are between 2 and $4[21,22]$. The transmitted power $P_{t}$ may be unknown or known. However, even though it is "known" it may contain uncertainties through calibration errors [11]. Note that if $\alpha=\beta=2$, (20) results in the standard free space path loss model. 


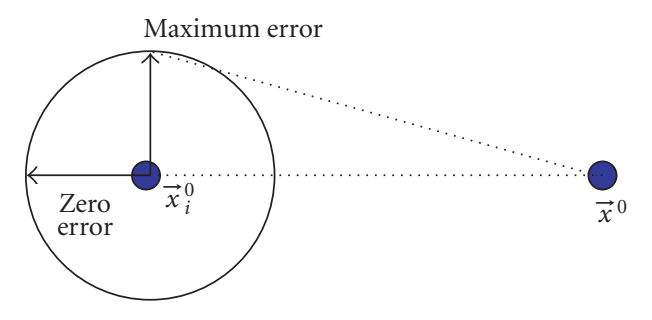

FIgURE 3: DOA estimation errors due to sensor location.

The propagation models predict the average (or median) received power level. However, real life channels may contain variations which may affect the received power level enormously $[11,23]$. The variations may lead to erroneous conclusions about the received power causing bias to the measurements. The channel variations may be compensated for by measuring the median of several consecutive measurements [23].

Since the system parameter vector

$$
\mathbf{q}=\left[\begin{array}{llllll}
P_{t} & \alpha & \beta & \mathbf{x}_{1}^{\mathrm{T}} & \cdots & \mathbf{x}_{N}^{\mathrm{T}}
\end{array}\right]^{\mathrm{T}},
$$

the $i$ th element on the measurement bias vector is

$$
\begin{gathered}
{\left[\mathbf{b}_{\mathbf{r}}\right]_{i}=P_{r_{i}}^{0}\left(\frac{\delta_{P_{t}}}{P_{t}^{0}}+\ln \left(\frac{d_{0}}{d_{i}^{0}}\right) \delta_{\alpha}+\ln \left(\frac{f_{0}}{f^{0}}\right) \delta_{\beta}\right.} \\
\left.+\alpha^{0} \frac{\left(\mathbf{x}^{0}-\mathbf{x}_{i}^{0}\right)^{\mathrm{T}} \delta_{\mathbf{x}_{i}}}{\left(d_{i}^{0}\right)^{2}}\right) .
\end{gathered}
$$

The assumed transmitted power $P_{t}=P_{t}^{0}+\delta_{P_{t}}$. If $P_{t}$ is much smaller than the actual power $\left(P_{t} \ll P_{t}^{0}\right)$, then $\delta_{P_{t}} / P_{t}^{0} \approx 1$ and the first term in (22) causes a measurement bias that is on the order of the actual power. If the assumed power is much larger than the actual power $\left(P_{t} \gg P_{t}^{0}\right)$, then $\delta_{P_{t}} / P_{t}^{0} \gg 1$ and the bias may be even much larger than the actual power. Since it is difficult to accurately guess the transmitted power of an unknown transmitter, it follows that the received signal strength location method does not very well suit those cases. If the transmitted power is known, then this measurement bias term can be neglected. However, even rather small uncertainties may cause severe effects. For example, if the uncertainty is $1 \mathrm{~dB}$, then $\delta_{P_{t}} / P_{t}^{0}=0.25$, which causes a significant measurement bias, of order $0.25 P_{r_{r}}^{0}$.

The second and third terms in (22) are then considered. The path loss attenuation factor errors $\delta_{\alpha}$ and $\delta_{\beta}$ may vary between -2 and 2 . However, the logarithms are small if the reference distance and frequency are selected small with respect to the actual distance and frequency, that is, if $d_{0} \ll d^{0}$ and $f_{0} \ll f^{0}$. Therefore, the effects of these two terms to the measurement errors are typically rather small.

The fourth term in (22) can be evaluated as the corresponding terms in the previous analyses. Therefore, the maximum bias through this term is $\alpha^{0}\left\|\delta_{\mathbf{x}_{i}}\right\| P_{r_{i}}^{0} / d_{i}^{0}$, which typically is rather small.
The RSS method is sensitive to the systematic errors also in another way. To see this, note that the Jacobian

$$
J_{\mathbf{x}}=-\alpha^{0} P_{t}^{0} d_{0}^{\alpha^{0}}\left(\frac{c}{4 \pi f_{0} d_{0}}\right)^{2}\left(\frac{f_{0}}{f^{0}}\right)^{\beta^{0}}\left[\begin{array}{c}
\frac{\left(\mathbf{x}^{0}-\mathbf{x}_{1}^{0}\right)^{\mathrm{T}}}{\left(d_{1}^{0}\right)^{\alpha^{0}+2}} \\
\vdots \\
\frac{\left(\mathbf{x}^{0}-\mathbf{x}_{N}^{0}\right)^{\mathrm{T}}}{\left(d_{N}^{0}\right)^{\alpha^{0}+2}}
\end{array}\right]
$$

depends on the propagation model. Therefore, also the estimated location depends on the propagation model and if there are errors on that, also the estimated location will be incorrect, that is, the actual mean-squared error cannot be predicted by the above method. To see this more clearly observe that the location may be computed iteratively (4) as

$$
\mathbf{x}_{k}=\mathbf{x}_{k-1}+\left(\mathbf{J}_{\mathbf{x}_{k-1}}^{\mathrm{T}} \mathbf{J}_{\mathbf{x}_{k-1}}\right)^{-1} \mathbf{J}_{\mathbf{x}_{k-1}}^{\mathrm{T}} \mathbf{e}_{k} .
$$

Since the Jacobian is dependent on the propagation model, a general comparison to the other methods is difficult. However, some conclusions can be made assuming the received powers are equal, say $P_{r}^{0}$. Then $J_{\mathbf{x}}=-\alpha^{0} P_{r}^{0} \mathbf{J}_{\mathrm{RSS}}$, where the rows of $\mathbf{J}_{\text {RSS }}$ are $\left(\mathbf{x}^{0}-\mathbf{x}_{i}^{0}\right)^{\mathrm{T}} /\left(d_{i}^{0}\right)^{2}$. Now the covariance becomes upper bounded as $n\left(\mathbf{J}_{\mathrm{RSS}}^{\mathrm{T}} \mathbf{J}_{\mathrm{RSS}}\right)^{-1}\left(\left(\sigma_{e}^{2}+b^{2}\right) /\left(\alpha^{0} P_{r}^{0}\right)^{2}\right)$, and comparison to other methods is possible if the estimation accuracy $\sigma_{e}^{2}$ and bias $b$ are known with respect to $P_{r}^{0}$. The term $\left(\mathbf{J}_{\mathrm{RSS}}^{\mathrm{T}} \mathbf{J}_{\mathrm{RSS}}\right)^{-1}$ is the approximated GDOP of the RSS method, which is rather similar to that of the TD method. Therefore, the RSS method may, at its best, perform like the TD method.

\subsection{Relative signal strength location}

RSS location systems were observed to be rather sensitive to uncertainties in the transmitted power. This sensitivity can be reduced using the relative RSS (RRSS) location method. Therein, two received powers are compared by dividing them such that the divider serves as a common reference. If the propagation loss model (20) is used and the $j$ th sensor is used as a reference, then the measurements of the RRSS method are modelled as

$$
r_{i}=\frac{P_{t}\left(c / 4 \pi f_{0} d_{0}\right)^{2}\left(f_{0} / f\right)^{\beta}\left(d_{0} / d_{i}\right)^{\alpha}+e_{i}}{P_{t}\left(c / 4 \pi f_{0} d_{0}\right)^{2}\left(f_{0} / f\right)^{\beta}\left(d_{0} / d_{j}\right)^{\alpha}+e_{j}},
$$

where the terms $e_{i}$ denote the measurement errors.

Since ideally the measurement errors are zero, the measurements become ideally as $r_{i}=\left(d_{j} / d_{i}\right)^{\alpha}$ and the $i$ th row of the Jacobian $\mathbf{J}_{\mathbf{x}}$ is

$$
\alpha^{0} \xi_{i}^{\alpha^{0}}\left[\frac{\left(\mathbf{x}^{0}-\mathbf{x}_{j}^{0}\right)^{\mathrm{T}}}{\left(d_{j}^{0}\right)^{2}}-\frac{\left(\mathbf{x}^{0}-\mathbf{x}_{i}^{0}\right)^{\mathrm{T}}}{\left(d_{i}^{0}\right)^{2}}\right],
$$

where $\xi_{i}=d_{j}^{0} / d_{i}^{0}$. Note that the ideal measurements do not depend on the transmitted power or the frequency and, thus, $\beta$. Therefore, the system parameters are

$$
\mathbf{q}=\left[\begin{array}{llll}
\alpha & \mathbf{x}_{1}^{\mathrm{T}} & \cdots & \mathbf{x}_{N}^{\mathrm{T}}
\end{array}\right]^{\mathrm{T}} .
$$


Due to the fact that the derivative of $a^{-x}$ with respect to $x$ is $-a^{-x} \ln a$, the $i$ th measurements bias term is

$$
\begin{aligned}
{\left[\mathbf{b}_{\mathbf{r}}\right]_{i}=} & \left(\xi_{i}^{\alpha^{0}} \ln \xi_{i}\right) \delta_{\alpha}+\frac{\alpha^{0} \xi_{i}^{\alpha^{0}}\left(\mathbf{x}^{0}-\mathbf{x}_{i}^{0}\right)^{\mathrm{T}} \delta_{\mathbf{x}_{i}}}{\left(d_{i}^{0}\right)^{2}} \\
& -\frac{\alpha^{0} \xi_{i}^{\alpha^{0}}\left(\mathbf{x}^{0}-\mathbf{x}_{j}^{0}\right)^{\mathrm{T}} \delta_{\mathbf{x}_{j}}}{\left(d_{j}^{0}\right)^{2}} .
\end{aligned}
$$

The last two terms in (28) are due to the sensor positioning errors. Their magnitudes are upper bounded by $\left(\xi_{i}^{0} \alpha^{0} /\right.$ $\left.d_{i}^{0}\right)\left\|\delta_{\mathbf{x}_{i}}\right\|$ and $\left(\xi_{i}^{0} \alpha^{0} / d_{i}^{0}\right)\left\|\delta_{\mathbf{x}_{j}}\right\|$. These terms are typically relatively small. The first term in (28) is due to channel modelling errors. It approaches zero if the reference sensor is much closer to the emitter than the other sensor, that is, $\xi_{i} \ll 1$. Therefore, this term may be relatively small. However, in the opposite case $\left(\xi_{i} \gg 1\right)$ it is large. As a conclusion, the measurements in the RRSS method are not sensitive to channel modelling errors assuming that all the measured channels obey the equal channel propagation loss law and that the closest sensor to the emitter is selected as a reference. As in the RSS method, the Jacobian of the RRSS method depends on the propagation model (through $\alpha$ ) and similar conclusions about the location hold.

\subsubsection{Measurement error covariance}

The Cramér-Rao bound for the RRSS method may be difficult to evaluate since the measurement model (25) involves the division of random variables. Therefore, the measurements are linearized with respect to measurement errors $e_{i}$ [15]. It follows that the measurement error covariance matrix becomes

$$
\mathrm{C}_{\mathrm{e}}=J_{\mathrm{e}} \overline{\mathbf{C}}_{\mathrm{e}} J_{\mathrm{e}}^{\mathrm{T}} \text {, }
$$

where $J_{\mathrm{e}}$ is the Jacobian and $\overline{\mathbf{C}}_{\mathbf{e}}$ is the covariance of measurement errors $e_{i}$. The $i$ th row of the Jacobian contains zeros except that the $i$ th element is $\partial r_{i} / \partial e_{i}=1 / P_{r_{j}}^{0}$ and the $j$ th element is $\partial r_{i} / \partial e_{j}=-P_{r_{i}}^{0} /\left(P_{r_{j}}^{0}\right)^{2}$. It follows that the diagonal elements of $\mathbf{C}_{\mathbf{e}}$ are

$$
\left[\mathbf{C}_{\mathbf{e}}\right]_{i i}=\frac{1}{\left(P_{r_{j}}^{0}\right)^{2}}\left[\delta_{e_{i}}^{2}+\left(\frac{P_{r_{i}}^{0}}{P_{r_{j}}^{0}}\right)^{2} \delta_{e_{j}}^{2}\right],
$$

where $\delta_{e_{i}}^{2}$ is the variance of the power measurement $P_{r_{i}}$. The error covariance (30) is minimized using the closest sensor to the emitter as a reference since the closest sensor provides the highest actual power.

\section{NUMERICAL RESULTS}

Early in this work the measurement covariance and especially the bias have been discussed. In Section 2 these were (approximately) shown to be directly related to location accuracy through the GDOP. Therefore, it suffices to consider the GDOP, or $\operatorname{tr}\{\mathbf{Q}\}$, as an additional performance measure of the location systems. The final accuracy is obtained by multiplying the GDOP values by the measurement error variance

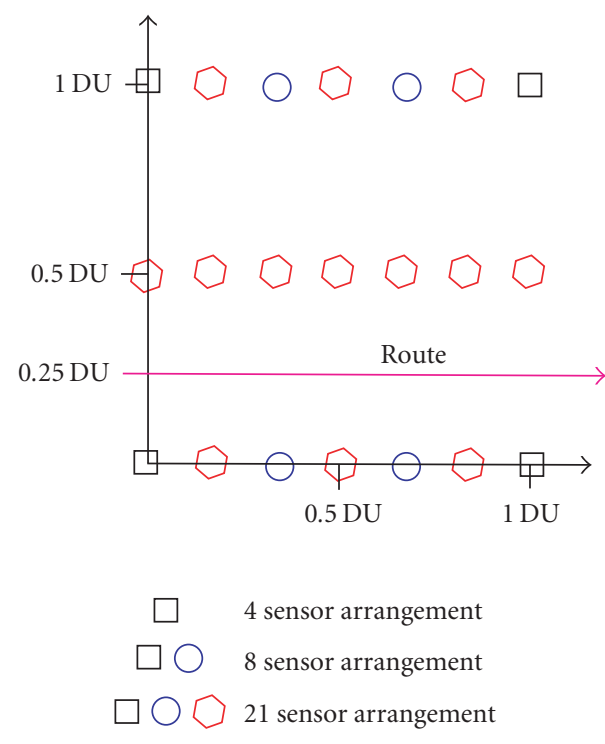

FIGURE 4: The sensor placements in different arrangements and the route of the emitter.

and bias and taking adequate distance scales into account, as will be soon discussed.

We present some numerical examples related to TD, DF, and RRSS location systems with 2D positioning. These serve just as illustrative examples but, still, provide some light to the accuracy problem. The examined sensor system is formed from 4,8 , or 21 sensors covering a square with the length 1 distance unit (DU) per side. The arrangement is shown in Figure 4. One DU can be any distance, for example, a meter, a kilometer, or $30 \mathrm{~km}$. Therefore, the shown results can be used to approximate geometric effects of different location systems with different sizes. The route of the emitter is also shown in the figure. The sensor at $(0,0)$ DU is selected as a reference if it is required. In what follows, the GDOP values are presented according to the horizontal axis of the route.

The results for the 4,8, and 21 sensor arrangements are illustrated in Figures 5, 6, and 7 for $\alpha=2$ (required in the RRSS). The results show that the RRSS method is rather sensitive to the selection of the reference sensor in the few sensor case. In contrast to the bias and measurement error analysis, the GDOP is large (even huge) if the closest sensor to the emitter is selected as a reference. Therefore, the bias and measurement accuracy yield different requirements on the reference sensor than the geometric effects. The RRSS method may, however, offer the lowest GDOP values if the reference sensor is selected appropriately. The DF method has the second best GDOP and the TD method has the worst GDOP. It can also be concluded that in dense networks it is not wise to select the furthest sensor from the emitter as a reference in the TD method. The DF method does not need a reference sensor and provides similar GDOP in all investigated positions.

The obtained GDOP values may be used as "rule of thumb" values in location accuracy studies. These should be multiplied by the variance, bias, and, in addition, the distance 


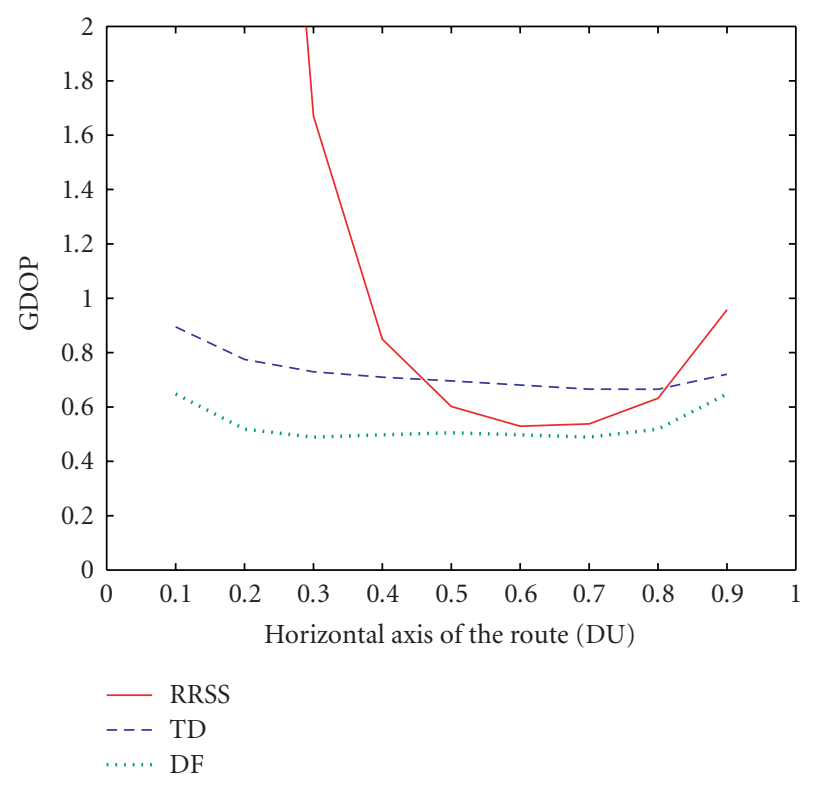

FIgURE 5: The GDOP in the 4 sensor case as a function of the emitter location with respect to the horizontal axis.

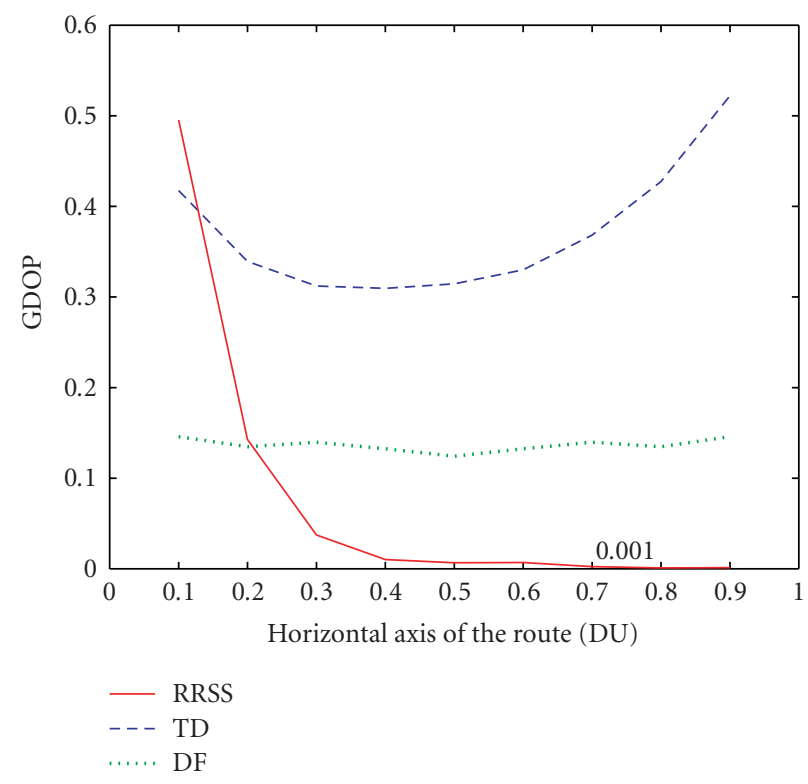

FIGURE 6: The GDOP in the 8 sensor case as a function of the emitter location with respect to the horizontal axis.

scale to attain the final location accuracy. The distance scale follows from the unit of the GDOP. The units of the Jacobians of the TD, DF, and RSS methods are unitless, $\mathrm{rad} / \mathrm{DU}$, and PU/DU, respectively, where PU stands for power unit. The corresponding units of the GDOP are unitless, $\mathrm{DU}^{2} / \mathrm{rad}^{2}$, and $\mathrm{DU}^{2} / \mathrm{PU}^{2}$. Therefore, the location accuracy of the $\mathrm{DF}$ and RRSS methods depends on the dimension of the net-

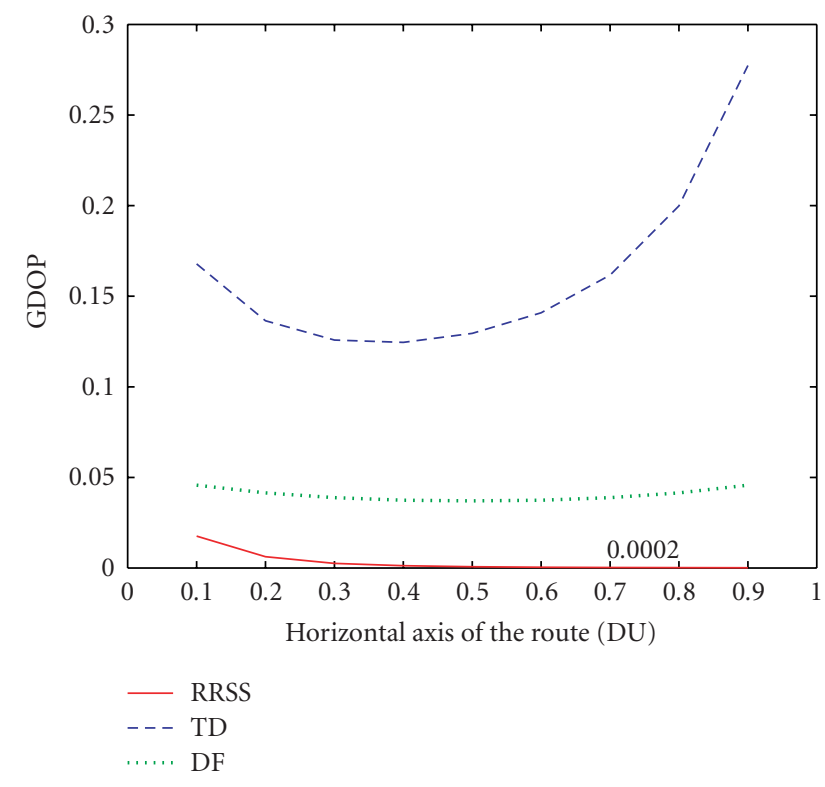

FIGURE 7: The GDOP in the 21 sensor case as a function of the emitter location with respect to the horizontal axis.

work, whereas the performance of the TD method does not (see also [11] for a discussion on GDOP units). An example will illustrate this. Typical measurement accuracies are presented in [10]. Using those we have $\sqrt{b^{2}+\sigma_{e}^{2}}$ values for TD, DF, and RRSS methods as $20 \mathrm{~m}, 6$ degrees, and $10 P_{r}^{0}$, respectively. If the DU is $10 \mathrm{~km}$, the root mean-squared location errors are obtained by multiplying the given accuracy with the square root of the GDOP value and by $20 \mathrm{~m}$ in the TD method, by $6 \pi / 180$ radians $\times 10 \mathrm{~km}$ in the DF method, and by $100 P U \times 10 \mathrm{~km}$ in the RRSS method.

It follows that the root mean-squared accuracies are $6.9 \mathrm{~m}$ for the TD method, $209.4 \mathrm{~m}$ for the DF method, and $1400 \mathrm{~m}$ for the RRSS method in the 21 sensor case. The TD method may therefore be preferred in large sized networks. In small sized networks the other methods may offer better performance than the TD method. In order to see this assume that the DU is $100 \mathrm{~m}$. Then the corresponding accuracies become $2.4 \mathrm{~m}, 2.1 \mathrm{~m}$, and $14 \mathrm{~m}$.

\section{CONCLUSIONS}

The effects of systematic errors on the location accuracy have been studied in this paper. A unified tool that can be used to analyze also the effects of measurement errors was introduced. It was analytically shown that our expectations on sensitivity are valid; the hyperbolic (time-delay-based) location methods are sensitive to errors in the network synchronization, the direction finding location methods are rather insensitive to systematic errors, and the received signals strength methods are rather sensitive to propagation loss modelling errors. The proposed analysis tool may be used to evaluate the magnitude of the location error through systematic errors. 
The relative received signal strength methods, which are less sensitive to the propagation model errors than the regular signal strength methods, are sensitive to the selection of the reference sensor. The furthest sensor from the emitter should be selected as a reference although this maximizes the effects of bias.

It was also shown that the hyperbolic methods may be preferred in large sized networks whereas the direction finding and received signal strength methods may offer better or adequate performance in small sized networks. The RSS methods usually require a dense sensor network in order to provide results that are related to the accuracy of the hyperbolic and direction finding methods; see $[11,12]$ for similar conclusions.

\section{APPENDIX}

For simplicity, let $\mathbf{J}=\mathbf{J}_{\mathbf{x}}\left(\mathbf{x}^{0}, \mathbf{q}^{0}\right)$. Consider the difference

$$
\begin{aligned}
\chi & =\mathbf{y}^{\mathrm{T}}\left[\left(\mathbf{J}^{\mathrm{T}} \mathbf{J}\right)^{-1} \mathbf{J}^{\mathrm{T}} a \mathbf{J}\left(\mathbf{J}^{\mathrm{T}} \mathbf{J}\right)^{-1}-\left(\mathbf{J}^{\mathrm{T}} \mathbf{J}\right)^{-1} \mathbf{J}^{\mathrm{T}} \mathbf{C}_{\mathbf{e}} \mathbf{J}\left(\mathbf{J}^{\mathrm{T}} \mathbf{J}\right)^{-1}\right] \mathbf{y} \\
& =\mathbf{y}^{\mathrm{T}}\left[\left(\mathbf{J}^{\mathrm{T}} \mathbf{J}\right)^{-1} \mathbf{J}^{\mathrm{T}}\left(a \mathbf{I}-\mathbf{C}_{\mathbf{e}}\right) \mathbf{J}\left(\mathbf{J}^{\mathrm{T}} \mathbf{J}\right)^{-1}\right] \mathbf{y},
\end{aligned}
$$

where $\mathbf{I}$ is an identity matrix, $\mathbf{y}$ is an arbitrary nonzero real vector, and $a$ is a real scalar. The eigendecomposition $\mathrm{C}_{\mathrm{e}}=$ $\mathrm{UDU}^{\mathrm{T}}$, where $\mathrm{D}$ is the diagonal matrix of the eigenvalues and $\mathbf{U}$ the matrix of eigenvectors, and the fact $\mathbf{U U}^{\mathrm{T}}=\mathbf{I}$ yield

$$
\begin{aligned}
& \chi=\mathbf{y}^{\mathrm{T}}\left(\mathbf{J}^{\mathrm{T}} \mathbf{J}\right)^{-1} \mathbf{J}^{\mathrm{T}}\left(a \mathbf{U} \mathbf{U}^{\mathrm{T}}-\mathbf{U D U} \mathbf{U}^{\mathrm{T}}\right) \mathbf{J}\left(\mathbf{J}^{\mathrm{T}} \mathbf{J}\right)^{-1} \mathbf{y} \\
& =\mathbf{y}^{\mathrm{T}}\left(\mathbf{J}^{\mathrm{T}} \mathbf{J}\right)^{-1} \mathbf{J}^{\mathrm{T}} \mathbf{U}(a \mathbf{I}-\mathbf{D}) \underbrace{\mathbf{U}^{\mathrm{T}} \mathbf{J}\left(\mathbf{J}^{\mathrm{T}} \mathbf{J}\right)^{-1} \mathbf{y}}_{\mathbf{z}} \\
& =\mathbf{z}^{\mathrm{T}}(a \mathbf{I}-\mathbf{D}) \mathbf{z} .
\end{aligned}
$$

It has been shown [24, page 142] that the eigenvalues of $\mathbf{C}_{\mathbf{e}}$ are upper bounded by $\left\|\left|\mathbf{C}_{\mathrm{e}}\right|\right\|_{\infty}$, the maximum absolute row sum norm. Since $\left\|\left|\mathbf{C}_{\mathrm{e}}\right|\right\|_{\infty} \leq n \sigma_{e}^{2}$, where $n$ is the dimension of $\mathrm{C}_{\mathrm{e}}$ and $\sigma_{e}^{2}$ the maximum element of it, it follows that $\chi \geq 0$ if $a=n \sigma_{e}^{2}$. This means that $n \sigma_{e}^{2}\left(\mathbf{J}^{\mathrm{T}} \mathbf{J}\right)^{-1} \geq\left(\mathbf{J}^{\mathrm{T}} \mathbf{J}\right)^{-1} \mathbf{J}^{\mathrm{T}} \mathbf{C}_{\mathrm{e}} \mathbf{J}\left(\mathbf{J}^{\mathrm{T}} \mathbf{J}\right)^{-1}$ in the sense that the difference of the matrices is positive definite. This concludes the proof.

The proof also shows that a tighter upper bound is obtained if the maximum eigenvalue of $\mathbf{C}_{\mathrm{e}}$ is chosen as $a$. Correspondingly, if the minimum eigenvalue of $\mathbf{C}_{\boldsymbol{e}}$ is chosen as $a$, the lower bound is obtained.

\section{ACKNOWLEDGMENTS}

This work has been supported by Finnish Defence Forces and is produced in the Finnish Software Radio Programme. We also like to thank our colleague Zach Shelby for his help in preparing the final manuscript.

\section{REFERENCES}

[1] IEEE Signal Processing Magazine, "Location is everything: positioning in wireless networks (a special issue)," IEEE Signal Processing Magazine, vol. 22, no. 3, 2005.
[2] B. M. Sadler, "Fundamentals of energy-constrained sensor network systems," IEEE Aerospace and Electronic Systems Magazine, vol. 20, no. 8, pp. 17-35, 2005, part 2: tutorials.

[3] W. H. Foy, "Position-location solutions by Taylor-series estimation," IEEE Transactions on Aerospace and Electronic Systems, vol. 12, no. 2, pp. 187-193, 1976.

[4] D. J. Torrieri, "Statistical theory of passive location systems," IEEE Transactions on Aerospace and Electronic Systems, vol. 20, no. 2, pp. 183-198, 1984.

[5] D. Kaplan, Understanding GPS Principles and Applications, Artech House, Boston, Mass, USA, 1996.

[6] N. Patwari, A. O. Hero III, M. Perkins, N. S. Correal, and R. J. O'Dea, "Relative location estimation in wireless sensor networks," IEEE Transactions on Signal Processing, vol. 51, no. 8, pp. 2137-2148, 2003.

[7] A. J. Weiss, "On the accuracy of a cellular location system based on RSS measurements," IEEE Transactions on Vehicular Technology, vol. 52, no. 6, pp. 1508-1518, 2003.

[8] S. Slijepcevic, S. Megerian, and M. Potkonjak, "Location errors in wireless embedded sensor networks: sources, models, and effect on applications," Mobile Computing and Communications Review, vol. 6, no. 3, pp. 67-78, 2002.

[9] A. H. Sayed, A. Tarighat, and N. Khajehnouri, "Network-based wireless location: challenges faced in developing techniques for accurate wireless location information," IEEE Signal Processing Magazine, vol. 22, no. 4, pp. 24-40, 2005.

[10] F. Gustafsson and F. Gunnarsson, "Mobile positioning using wireless networks: possibilities and fundamental limitations based on available wireless network measurements," IEEE Signal Processing Magazine, vol. 22, no. 4, pp. 41-53, 2005.

[11] N. Patwari, J. N. Ash, S. Kyperountas, A. O. Hero III, R. L. Moses, and N. S. Correal, "Locating the nodes: cooperative localization in wireless sensor networks," IEEE Signal Processing Magazine, vol. 22, no. 4, pp. 54-69, 2005.

[12] T. Pham, B. Sadler, and H. Papadopoulus, "Energy-based source localization via ad-hoc acoustic sensor network," in Proceedings of IEEE Workshop on Statistical Signal Processing, pp. 387-390, St. Louis, Mo, USA, September-October 2003.

[13] A. Savvides, W. L. Garber, R. L. Moses, and M. B. Srivastava, "An analysis of error inducing parameters in multihop sensor node localization," IEEE Transactions on Mobile Computing, vol. 4, no. 6, pp. 567-577, 2005.

[14] N. E. Wu and M. L. Fowler, "Aperture error mitigation via local-state estimation for frequency-based emitter location," IEEE Transactions on Aerospace and Electronic Systems, vol. 39, no. 2, pp. 414-429, 2003.

[15] J. R. Taylor, An Introduction to Error Analysis: The Study of Uncertainties in Physical Measurements, University Science Books, Sausalito, Calif, USA, 2nd edition, 1997.

[16] S. Gezici, Z. Tian, G. B. Giannakis, et al., "Localization via ultra-wideband radios: a look at positioning aspects of future sensor networks," IEEE Signal Processing Magazine, vol. 22, no. 4, pp. 70-84, 2005.

[17] A. E. Khuri, Advanced Calculus with Applications in Statistics, John Wiley \& Sons, New York, NY, USA, 1993.

[18] G. C. Carter, "Time delay estimation for passive sonar signal processing," IEEE Transactions on Acoustics, Speech, and Signal Processing, vol. 29, no. 3, pp. 463-470, 1981.

[19] X. Wang, Z. Wang, and B. O’Dea, "A TOA-based location algorithm reducing the errors due to non-line-of-sight (NLOS) propagation," IEEE Transactions on Vehicular Technology, vol. 52, no. 1, pp. 112-116, 2003. 
[20] S. M. Kay, Fundamendals of Statistical Signal Prosessing: Estimation Theory, PTR Prentice Hall, Englewood Cliffs, NJ, USA, 1993.

[21] H. H. Xia, "Simplified analytical model for predicting path loss in urban and suburban environments," IEEE Transactions on Vehicular Technology, vol. 46, no. 4, pp. 1040-1046, 1997.

[22] T. K. Sarkar, Z. Ji, K. Kim, A. Medouri, and M. Salazar-Palma, "A survey of various propagation models for mobile communication," IEEE Antennas and Propagation Magazine, vol. 45, no. 3, pp. 51-82, 2003.

[23] W. G. Figel, N. H. Shepherd, and W. F. Trammell, "Vehicle location by a signal attenuation method," IEEE Transactions on Vehicular Technology, vol. 18, no. 3, pp. 105-109, 1969.

[24] R. A. Horn and C. R. Johnson, Topics in Matrix Analysis, Cambridge University Press, Cambridge, UK, 1991.

Harri Saarnisaari received the M.S. degree in applied electrical physics from the University of Kuopio in 1993 and the Ph.D. degree in digital communications from the University of Oulu in 2000. Since 1994 he has been working as a teacher, researcher, and project manager at Telecommunications Laboratory and Centre for Wireless Communications (CWC) at the University of Oulu. Currently he is a Senior Research Scientist in CWC but also gives graduate and postgraduate courses. His research interest lies in signal processing. Especially he is interested in direct-sequence code phase synchronization in hostile environment, adaptive antenna algorithms, and channel estimation and positioning. He has been participating in the design and development of advanced software-defined radio for Finnish defence forces.

Timo Bräysy received his M.S. degree in 1991 and his Ph.D. degree in 2000, both in physics from the University of Oulu, Finland. He has a wide experience in scientific research in space physics and has worked in satellite instrument and software development projects. From 2000 to 2001 he worked as a Research Scientist in the Wireless Communications Group in VTT Electronics, Finland. Currently he is a researcher

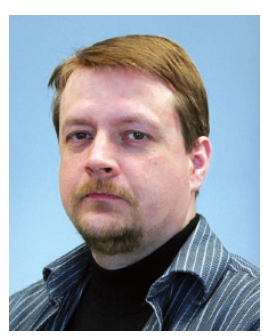
and project manager in Centre for Wireless Communications, University of Oulu. His current research interests include multiple access and networking protocols in mobile wireless networks with special emphasis on constraints set by security and defense application areas. 